\title{
Galvanic Vestibular Stimulation Induces a Spatial Bias in Whole-body Position Estimates
}

Mitesh Patel1, R. Edward Roberts1, Qadeer Arshad1, Maroof Ahmed, Mohammed U. Riyaz, Adolfo M. Bronstein

Academic Department of Neuro-otology

Division of Brain Sciences

Charing Cross Hospital Campus

Imperial College London

Fulham Palace Road, London W6 8RF, UK

* Corresponding author. Division of Brain Sciences, Department of Medicine, Imperial College London, Charing Cross Hospital, London W6 8RF, UK. Tel.: p44 7891161537.

E-mail address: q.arshad@imperial.ac.uk (Q. Arshad)

\section{Letter to the Editor}

Dear Editor,

Peripheral galvanic vestibular stimulation (GVS) has been shown to temporarily ameliorate left spatial neglect [1]. Specifically, anodal (facilitatory) stimulation over the left mastoid bone coupled with cathodal (inhibitory) over the right mastoid reduces visuospatial-neglect scores in line cancellation [2] and line bisection tasks [3,4]. This montage increases activity in the left vestibular nerve and suppresses activity in the right [5], which has been shown to focally activate vestibular networks that occupy visuospatial attention mechanisms, primarily in the non-dominant hemisphere [5]. Thus, it appears that electrical stimulation of the peripheral vestibular system can shift visuospatial attention to the left side of space [4]. However, whether such a shift of spatial attention in normal subjects can influence perception of spatial position during whole body spatial translations is unknown. We hypothesised that shifting attention to the left would result in participants underestimating spatial position estimates during rightward whole-body translations and overestimating spatial position estimates during leftward whole-body translations.

12 right-handed healthy males (mean age 21.6 years $(\mathrm{SD}=3.1)$ ) participated in this randomised cross-over study. Participants provided written informed consent. The study was approved by the local ethics committee. Participants were blindfolded and stood with their feet shoulderwidth apart upon a computer controlled linear sled, running on a level track along the interaural axis (Fig 1A). Participants were asked to stand upright with their head facing straight ahead and hold onto a sled-mounted support to minimise body movement - verified by trunk 
(C7) position recordings (Fastrak tracking system, Polhemus, VT, USA). Conductive electrode pads $(4 \mathrm{~cm} \times 5 \mathrm{~cm})$ were positioned over the right and left mastoid bones. DC Stimulation was delivered at $1 \mathrm{~mA}$ [4] either continually during sled movement 'continual stimulation' or for a single brief pulse at the onset of sled movement 'sham stimulation'. The stimulation conditions were: left anode / right cathode (LA/RC), right anode / left cathode (RA/LC) or sham, performed in a randomised order.

Each trial began with the sled at position zero (start) and then translated either leftward or rightward at a constant velocity of $0.3 \mathrm{~m} / \mathrm{s}$ [6], ramp up/ramp down time: $1.0 \mathrm{~s}$; well above translational thresholds [7]. Possible stopping positions were a series of five equally spaced positions beginning $1 \mathrm{~m}$ from the start position, labelled " 1 to 5 " separated by $50 \mathrm{~cm}$ intervals along the same path as the linear track, akin to a ruler on the floor (Figure 1A). Participants performed five practice runs through the positions 1-5 with visual feedback (raising the blindfold).

For each stimulation condition, participants were translated to each of the five possible stopping positions in each direction four times in a randomised order, giving a total of 40 trials (4 repeats $\mathrm{x} 5$ positions $\mathrm{x} 2$ directions) per stimulation condition (LA/RC, RA/LC or sham). For each trial participants estimated the position number they thought they had stopped at. To control for possible numerical biasing, we performed the same experiment using letters of the alphabet as markers (A-G) [8] in five naive right-handed males.

In each condition, we summed the error from each trial for each subject taking into account the polarity of each error as positive (overestimate) or negative (underestimate). The total error during the sham condition was subtracted from the total error in the galvanic condition, thus controlling for baseline performance.

As shown in Figure 1B, LA/RC GVS modulated perceived spatial position estimates in a direction specific manner. A $2 \times 2$ repeated-measures ANOVA, with factors stimulation side (2 levels: LA/RC, RA/LC) and translation direction (2 levels: right, left), revealed a significant main effect of direction $(F[1,11]=91.3 ; \mathrm{P}<0.001)$ but not stimulation side $(\mathrm{F}[1,11]=0.73 ; \mathrm{P}=0.41)$, and a significant Stimulation side $x$ Direction interaction $(F[1,11]=52.1 ; \mathrm{P}<0.001)$. That is, GVS influenced the polarity of errors for leftward and rightward translations. Post-hoc paired t-tests showed that LA/RC GVS produced a significantly smaller estimate of distance moved during rightward translations $(\mathrm{P}<0.001)$ and a significantly larger estimate during leftward translations $(\mathrm{P}<0.001)$ compared to RA/LC stimulation (Fig. 1B). The same pattern of responses was found in the alphabetic experiment (Direction effect $(\mathrm{F}[1,4]=18.4 ; \mathrm{P}<0.001)$; Stimulation side $x$ Direction interaction $(F[1,4]=9.5 ; P=0.02))$. 


\section{Figure 1 here}

We provide the first demonstration that LA/RC GVS shifts estimates of whole-body spatial position. A possible explanation for this finding is an altered gain of the peripheral vestibular system. However, for this explanation to hold we would expect to observe biases for both active stimulation conditions, which we do not. Eye movements could also have influenced position estimates. GVS at around $1 \mathrm{~mA}$ is known to produce both torsional and weak horizontal eye movements towards the anode [9]. However, the fact that we did not observe position biases in both active stimulation conditions also rules out the possibility of a gaze-shift mediated effect.

The most parsimonious explanation for our results is that LA/RC GVS biased spatial attention during the position task. Previous studies have shown that this montage biases visuospatial attention to the left space $[1,2,4]$. We show that when participants perform a whole body spatial position task, LA/RC stimulation induces a relative spatial bias towards left space which results in participants underestimating spatial position estimates during rightward whole-body translations, and overestimating spatial position estimates during leftward whole-body translations. Further, these biases are not secondary to numerical biasing as the alphabetical control experiment yielded identical results.

To conclude, our data indicates that LA/RC GVS induces hemispheric biases in spatial attentional networks which subsequently disrupts position estimates.

\section{Acknowledgements}

We are supported by the UK Medical Research Council (MR/J004685/1) and the National Institute for Health Research (NIHR) Imperial Biomedical Research Centre. We acknowledge the technical assistance from Mr David Buckwell (Imperial College London).

\section{References}

[1] D. Wilkinson, O. Zubko, M. Sakel, S. Coulton, T. Higgins, P. Pullicino. Galvanic vestibular stimulation in hemi-spatial neglect. Front Integr Neurosci 2014; 8: 4.

[2] I. Rorsman, M. Magnusson, B.B. Johansson. Reduction of visuo-spatial neglect with vestibular galvanic stimulation. Scand J Rehabil Med 1999; 31: 117-124.

[3] K.S. Utz, I. Keller, M. Kardinal, G. Kerkhoff. Galvanic vestibular stimulation reduces the pathological rightward line bisection error in neglect-a sham stimulation-controlled study. Neuropsychologia 2011; 49: 1219-1225.

[4] E.R. Ferre, M.R. Longo, F. Fiori, P. Haggard. Vestibular modulation of spatial perception. Front Hum Neurosci 2013; 7: 660. 
[5] G.R. Fink, J.C. Marshall, P.H. Weiss, T. Stephan, C. Grefkes, N.J. Shah, K. Zilles, M. Dieterich. Performing allocentric visuospatial judgments with induced distortion of the egocentric reference frame: an fMRI study with clinical implications. Neuroimage 2003; 20: 1505-1517.

[6] M. Hartmann, R. Farkas, F.W. Mast. Self-motion perception influences number processing: evidence from a parity task. Cogn Process 2012; 13 Suppl 1: S189-192.

[7] C. Gianna, S. Heimbrand, M. Gresty. Thresholds for detection of motion direction during passive lateral whole-body acceleration in normal subjects and patients with bilateral loss of labyrinthine function. Brain Res Bull 1996; 40: 443-447; discussion 448-449.

[8] M. Zorzi, K. Priftis, F. Meneghello, R. Marenzi, C. Umilta. The spatial representation of numerical and non-numerical sequences: evidence from neglect. Neuropsychologia 2006; 44: 1061-1067.

[9] A. Severac Cauquil, M. Faldon, K. Popov, B.L. Day, A.M. Bronstein. Short-latency eye movements evoked by near-threshold galvanic vestibular stimulation. Exp Brain Res 2003; 148: 414-418. 


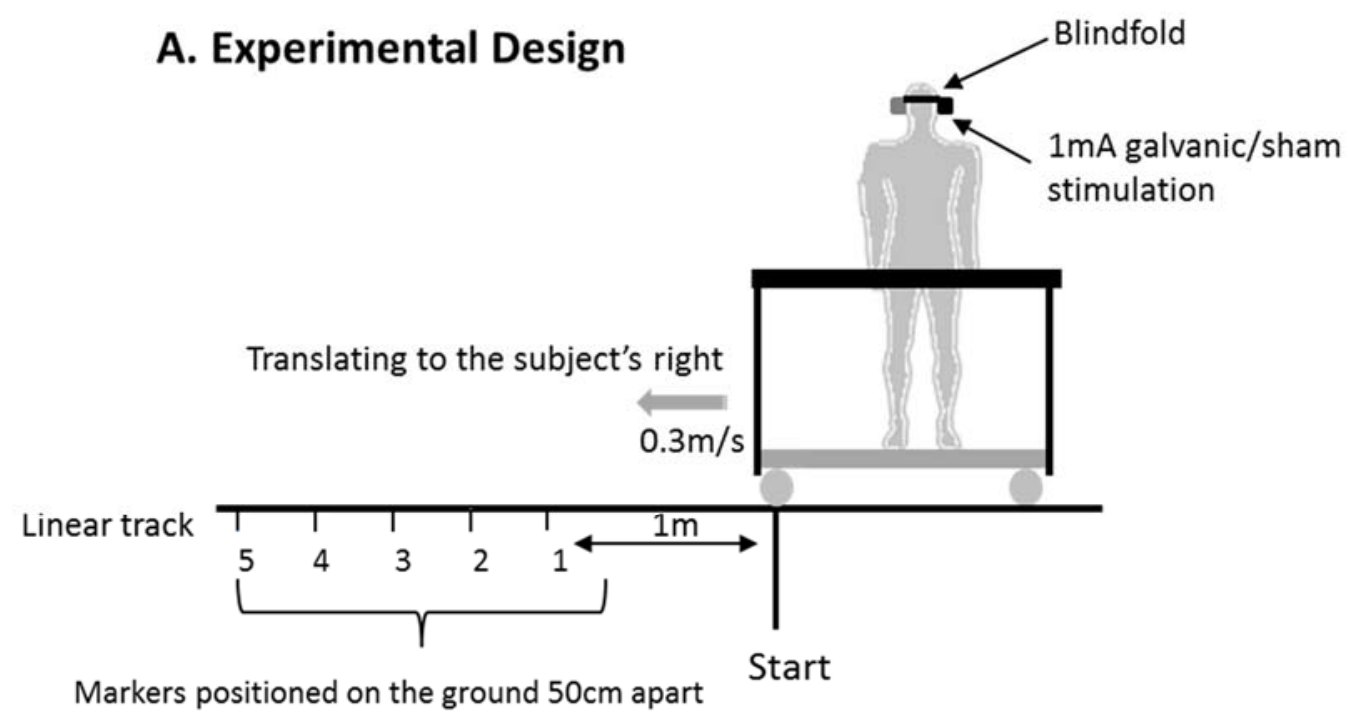

\section{B. Results}

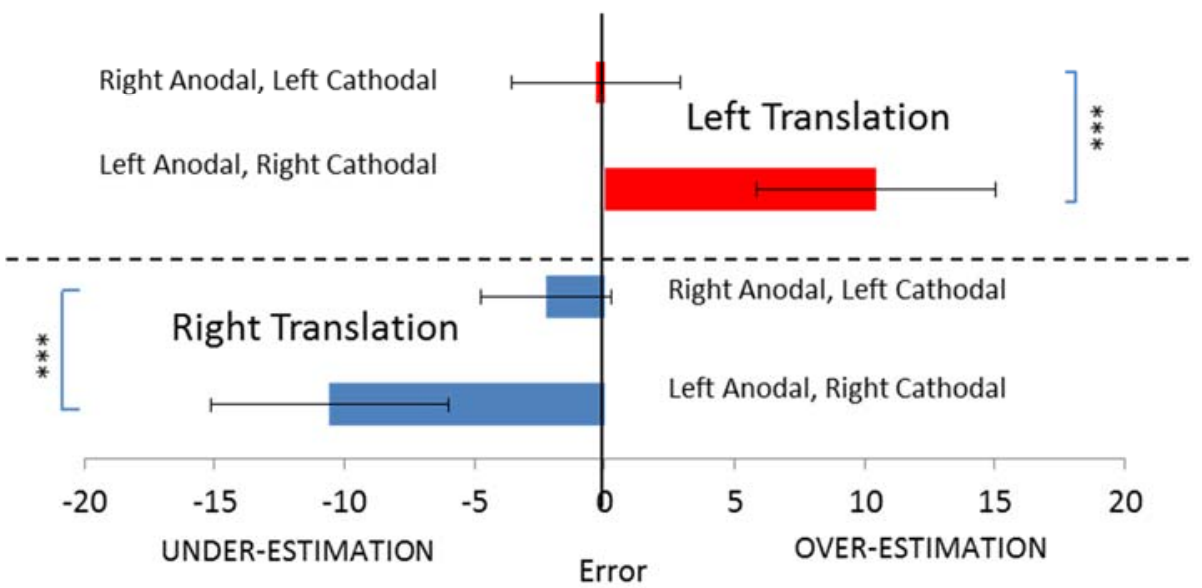

Figure 1 (A). Experimental Design. Subjects were blindfolded and stood on a linear sled. Electrode pads were fixed over the mastoid bones on the right and left side which delivered either RA/LC, LA/RC or sham galvanic stimulation. The sled was translated rightwards or leftwards and participants were asked to estimate how far they thought the sled had moved, providing a number in the numerical experiment or a letter in the alphabetic experiment. The figure represents a rightward translation.

(B). Mean error scores (+/-SD) for the numerical interval experiment. The numerical experiment showed that subjects underestimated the distance moved less for rightward translations and overestimated for leftward translations during left anodal/right cathodal stimulation. 